Pacific Journal of Mathematics

OPERATOR-VALUED INNER FUNCTIONS ANALYTIC ON THE 


\title{
OPERATOR-VALUED INNER FUNCTIONS ANALYTIC ON THE CLOSED DISC II
}

\author{
StePhen L. Campbell
}

An operator-valued inner function $V$ is called scalar if $\{V(w):|w|<1\}$ is a commuting family of normal operators. Suppose that $T$ is a bounded linear operator with $\|T\| \leqq 1$ and spectral radius strictly less than one. Let $V_{T}$ be its Potapov inner function and define $U_{T}=V_{T} V_{T}^{*}(1)$. The structure of nonnormal $T$ for which $U_{T}$ is scalar is discussed. An explicit characterization is given if the underlying Hilbert space is finite dimensional. Examples are given for the infinite dimensional case. The relationship between scalar inner functions and operators for which $T^{*} T$ and $T^{*}+T$ commute is examined.

1. Introduction. Sherman [9] introduced the concept of an inner function of scalar type. He observed that the Potapov inner function $V_{T}$ of a normal operator $T,\|T\|<1$, was of scalar type. On the other hand Campbell [1] has shown that if $\|T \phi\|<\|\phi\|$ for all nonzero vectors $\phi$, then $V_{T}$ is of scalar type if and only if $T$ is normal. There are, however, non-normal operators associated with scalar inner functions. It will be shown that if $T^{*} T$ and $T+T^{*}$ commute, then $T$ is the restriction of an operator $\tilde{T}$ such that $V_{\tilde{T}}$ differs from an inner function of scalar type by a constant unitary operator on the right. Thus, when studying the operators such that $T^{*} T$ and $T+T^{*}$ commute, it would be helpful to have information on operators with scalar inner functions and their invariant subspaces. We shall develop some of the needed information.

2. Terminology. We assume that the reader is familiar with the basic definitions of our terms. They may be found in [6]. Our notation is that of [1]. We review it briefly. Throughout this paper $h$ will be a fixed separable Hilbert space. $H_{h}^{2}$ will denote the $h$-valued Hardy space of the circle, $|w|=1 . \quad S$ is multiplication by $w$ in $H_{h}^{2} \quad T$ will always be a bounded linear operator from $h$ into $h$. If $\|T\| \leqq 1$, $T^{n} \rightarrow 0$ strongly, and $T^{*}$ is not an isometry, then the Potapov inner function $V_{T}$ of $T$ is defined by

$$
V_{T}(w)=-T^{*}+w \sum_{n=0}^{\infty}\left(I-T^{*} T\right)^{1 / 2} w^{n} T^{n}\left(I-T T^{*}\right)^{1 / 2}
$$


If the spectral radius of $T, r(T)$, is less than one, then (1) takes the form

$$
V_{T}(w)=-T^{*}+w\left(I-T^{*} T\right)^{1 / 2}(I-w T)^{-1}\left(I-T T^{*}\right)^{1 / 2}
$$

and $V_{T}$ is analytic on the closed disc $|w| \leqq 1$. If $\|T\|<1$, then (1) becomes

$$
V_{T}(w)=\left(I-T^{*} T\right)^{-1 / 2}\left(w I-T^{*}\right)(I-w T)^{-1}\left(I-T T^{*}\right)^{1 / 2}
$$

For an inner function $U$, we say that $U$ is an analytic inner function, $U \in(A I)$, if $U$ is analytic on the closed disc $|w| \leqq 1$. If $U \in(A I)$ and $U(1)=I$, the identity on $h$, we say $U$ is normalized. For any $U \in(A I)$, $U U^{*}(1)$ is called its normalized form. An inner function will be called scalar [9] if $U(w)$ commutes with $U(u)$ for almost all $|w|=|u|=$ 1. This is equivalent to having all the coefficients in the power series for $U$ commute.

If $U \in(A I)$, let $z=i(1-w) /(1+w)$ and define $\tilde{U}(z)=$ $U(w)$. Then $\tilde{U}$ is an inner function on the upper half-plane. Let $x, y$ denote real values of $z$. The variables $x, y, z, w$ will always be used in this manner. Now $\tilde{U}$ satisfies the differential equation $\tilde{U}^{\prime}(x)=$ $i A(x) \tilde{U}(x)$ where $A(x) \geqq 0$ and $A$ is analytic on a neighborhood of the real axis [7]. If $U=V_{T}$ for some $T, r(T)<1$, then we write $A_{T}$ for A. A straightforward calculation gives

$$
A_{T}(x)=\rho(x)\left(I-T^{*} T\right)^{1 / 2}(I-w T)^{-1}\left(w I-T^{*}\right)^{-1}\left(I-T^{*} T\right)^{1 / 2}
$$

where $\rho(x)$ is a scalar valued function which is always nonzero.

For bounded linear operators $X, Y$, let $[X, Y]=X Y-Y X$.

Finally, we let $\Theta=\left\{T:\left[T^{*} T, T+T^{*}\right]=0\right\}$.

3. Preliminary results. It is clear from (1) that if $V_{T}$ is scalar, then $\left[T, T^{*}\right]=0$ and $T$ is normal. Given a $T \in \Theta$, we shall construct in Section 8 a non-trivial extension $\tilde{T}$ of $T$ such that $V_{\tilde{T}} V_{\tilde{T}}^{*}(1)$ is scalar.

In order to try and understand the structure of these $T$, we will study those $V_{T}$ with scalar normalized form. But first, we shall show how our results relate to the structure of a general analytic inner function. Note that if $U \in(A I)$, the normalized form of $U$ is scalar if and only if there exists a constant unitary operator $U_{0}$ such that $U U_{0}$ is scalar.

PRoposition 1. Suppose that $U \in(A I)$. The normalized form of $U$ is scalar if and only if $[A(x), A(y)]=0$ for every real $x, y$.

Proof. The only if part follows by twice differentiating $U(x) U(y)=$ 
$U(y) U(x)$. On the other hand if $[A(x), A(y)]=0$ for every real $x, y$, then $W(x)=\exp \left(i \int_{0}^{x} A(s) d s\right)$ and the normalized form of $U$ both satisfy the differential equation $X^{\prime}=i A X, X(0)=I$, and hence are equal. But $W$ is scalar, hence the normalized form of $U$ is.

It was shown in $[1$, p. 58] that if $U \in(A I)$, then

$$
U=c V_{T} \tau U_{1} \oplus U_{2} \quad \text { where } \quad h=h_{1} \oplus h_{2} .
$$

Here $U_{i}$ is a constant unitary operator on $h_{i}, T$ is an operator from $h_{1}$ into $h_{1}$ such that $\|T\| \leqq 1, r(T)<1, \tau$ is an isometry from $h_{1}$ onto $R\left(\left[I-T T^{*}\right]^{1 / 2}\right)$, and $c^{*}$ is an isometry from $h_{1}$ onto $R\left(\left[I-T^{*} T\right]^{1 / 2}\right)$. Furthermore $A(x)=c A_{T}(x) c^{*} \oplus 0$. Using (4) and the definition of $c$ we see that $[A(x), A(y)]=0$ for all real $x$ and $y$ if and only if $\left[A_{T}(x), A_{T}(y)\right]=0$ for all real $x$ and $y$.

Thus as a consequence of Proposition 1 we have:

Proposition 2. If $U$ and $V_{T}$ are related as in (5), then the normalized form of $U$ and the normalized form of $V_{T}$ are either both scalar or both not scalar.

The determination of which normalized analytic inner functions are scalar reduces then to determining which analytic Potapov inner functions have a scalar normalized form. If $\|T\| \leqq 1, r(T)<1, T$ is not normal, and $V_{T}$ has scalar normalized form, then 1 is an eigenvalue of $T^{*} T[3$, Theorem 6].

We now turn to describing those $T$ for which the normalized form of $V_{T}$ is scalar. For notational convenience let $U_{T}(w)=V_{T}(w) V_{T}^{*}(1)$.

The next Proposition will be useful in what follows.

Proposition 3. Suppose that $\|T\| \leqq 1, r(T)<1$. Then $T$ has a reducing subspace $M \subseteq h$ if and only if $U_{T}=U_{1} \oplus U_{2}$ where $U_{i}$ is an inner function on $H_{h_{i}}^{2}, h=h_{1} \oplus h_{2}$.

Proof. If $T$ has a reducing subspace $M$, the result is obvious. Suppose then that $U_{T}=U_{1} \oplus U_{2}$ on $H_{h}^{2}=H_{h_{1}}^{2} \oplus H_{h_{2}}^{2}$. Now

$$
H_{h}^{2} \ominus\left(U_{T} H_{h}^{2}\right)=\left(H_{h_{1}}^{2} \ominus U_{1} H_{h_{1}}^{2}\right) \oplus\left(H_{h_{2}}^{2} \ominus U_{2} H_{h_{2}}^{2}\right) .
$$

But $H_{h_{i}}^{2} \ominus U_{i} H_{h_{1}}^{2}, \quad i=1,2$, is an invariant subspace for $S^{*}$ on $H_{h}^{2} \ominus U_{T} H_{h}^{2}$. Thus they are reducing subspaces. But $S^{*}$ restricted to $H_{h}^{2} \ominus U_{T} H_{\hbar}^{2}$ is unitarily equivalent to $T$. The unitary map $B$ from $h$ to $H_{h}^{2} \ominus U_{T} H_{h}^{2}$ is given by $B \phi=\left(I-T^{*} T\right)^{1 / 2}(I-w T)^{-1} \phi$. That is, $S^{*} B \phi=$ $B T \phi$ for every $\phi \in h$. Thus $M=B^{-1}\left(H_{h_{1}}^{2} \ominus U H_{h_{1}}^{2}\right)$ would be reducing for $T$. 
It is important to note that the $h_{1}$ of Proposition 3 may not be a reducing subspace of $T$. However, as observed in the proof of Proposition 3, $M_{i}=B^{-1}\left(H_{h_{i}}^{2} \ominus U_{i} H_{h_{i}}^{2}\right)$ is reducing for $T$. Thus $T=T_{1} \oplus T_{2}$ on $M_{1} \oplus M_{2}$. But then $U_{T}=U_{T_{1}} \oplus U_{T_{2}}$, and

$$
B M_{i}=H_{M_{i}}^{2} \ominus U_{T_{i}} H_{M_{i}}^{2}=H_{h_{i}}^{2} \ominus U_{i} H_{h_{i}}^{2} .
$$

If one assumes that $U_{1}^{\prime}(x=0)$ is one to one on $h_{1}$, then $h_{1} \subseteq M_{1}$ and $U_{T_{1}}$ is the identity on $M_{1} \ominus h_{1}$ since $U_{1}^{\prime}(x=0)$ being one-to-one implies that $U_{1}$ does not have a constant summand [2]. But $U_{T_{1}} \phi=\phi$ implies that $\left(I-T_{1}^{*} T_{1}\right) \phi=0$. We summarize these observations in the next theorem.

THEOREM 1. Suppose that $U_{T}=U_{1} \oplus U_{2}$ on $H_{h_{1}}^{2} \oplus H_{h_{2}}^{2}, \quad h=$ $h_{1} \oplus h_{2}$. Suppose further that $U_{1}^{\prime}(x=0)$ is one-to-one. Then $T$ has a reducing subspace $M$ such that $h_{1} \subseteq M$ and $M \ominus h_{1} \subseteq N\left(\left[I-T^{*} T\right]\right)$.

4. The finite dimensional case. Suppose that $r(T)<1$. If $U_{T}$ is scalar and $\operatorname{dim} h=n<\infty$, then $U_{T}$ is unitarily equivalent to an $n \times n$ diagonal matrix, $\operatorname{Diag}\left\{b_{1}, \cdots, b_{r}, 1, \cdots, 1\right\}$, where $b_{i}$ is a finite Blaschke product times a complex number of modulus one. We can now apply Theorem 1 to get:

THEOREM 2. Suppose that $\|T\|<1, \quad r(T)<1, \quad$ and $\operatorname{dim} h<$ $\infty$. Then $U_{T}$ is scalar if and only if $T=\sum_{i=1}^{r} \oplus T_{i}$ on $h=\sum_{i=1}^{r} \oplus h_{i}$ where $\operatorname{rank}\left(I-T_{i}^{*} T_{i}\right)=1$.

Note that the $T_{i}$ of Theorem 2 are unitarily equivalent to $S^{*}$ restricted to $H^{2} \ominus b H^{2}$ where $b$ is a scalar inner function. A related study may be found in [10]. Sickler, however, is primarily interested in the case $r(T)=1$ and his results do not overlap ours. The following characterization of the $T_{i}$ in Theorem 2 follows fom the observation that $T$ is an isometry on $N\left(I-T^{*} T\right)$.

Proposition 4. Suppose that $\operatorname{dim} h=n<\infty$ and $\|T\| \leqq 1$. Then $\operatorname{rank}\left(I-T^{*} T\right)=1$ if and only if $T=\delta V+W$ where $\delta$ is a scalar, $0 \leqq|\delta|<1$, and $V, W$ are partial isometries such that; rank $V=1$, rank $W=n-1, V^{*} W=0$, and $V W^{*}=0$.

5. The general case. In [9] Sherman showed that if $U$ is scalar, then $U(w)=\int_{0}^{2 \pi} f(w, \lambda) d E(\lambda),|w| \leqq 1$, for a particular spectral measure $E(\cdot)$ on the unit circle. An easy modification of his arguments shows that if $U \in(A I)$ and $U$ is scalar, then $f(w, \lambda)$ is a finite Blaschke 
product for almost all $\lambda$. One can also conclude that the zeros of $f(w, \lambda)$ for different $\lambda$ are essentially bounded away from $|w|=1$. However, the order and number of the zeros may be arbitrarily large. (Just take $T$ to be the orthogonal sum of the appropriate operators acting in finite dimensional spaces.) One could then argue that if $\|T\| \leqq 1, r(T)<1$, and $U_{T}$ is scalar, then $T$ is an integral (or orthogonal sum if $E(\cdot)$ is a discrete measure) of operators $T_{\lambda}$ where $T_{\lambda}$ acts in a finite dimensional space and is of the form described in the previous section.

There is one weakness with this approach. Using $T \in \Theta$ it is possible to construct operators $\tilde{T}$ such that $\|\tilde{T}\| \leqq 1, r(\tilde{T})<1$, and $U_{\tilde{T}}$ is scalar, but $\tilde{T}$ does not appear to be like the finite dimensional case.

First, we wish to give some additional examples of scalar $U_{T}$. A way of testing a $T$ to see whether $U_{T}$ is scalar is needed. We begin by calculating the coefficients of $U_{T}$. From (1) and (2) we have that the constant term is

$$
\begin{aligned}
& T^{*} T-T^{*}\left(I-T T^{*}\right)^{1 / 2}\left(I-T^{*}\right)^{-1}\left(I-T^{*} T\right)^{1 / 2} \\
& \begin{aligned}
(6)= & T^{*} T-\left(I-T^{*} T\right)^{1 / 2} T^{*}\left(I-T^{*}\right)^{-1}\left(I-T^{*} T\right)^{1 / 2} \\
= & T^{*} T-\left(I-T^{*} T\right)^{1 / 2}\left\{\left(I-T^{*}\right)^{-1}-I\right\}\left(I-T^{*} T\right)^{1 / 2} \\
& =I-\left(I-T^{*} T\right)^{1 / 2}\left(I-T^{*}\right)^{-1}\left(I-T^{*} T\right)^{1 / 2} .
\end{aligned}
\end{aligned}
$$

The $(n+1)$-th term, $n \geqq 0$, is

$$
\begin{aligned}
& -\left(I-T^{*} T\right)^{1 / 2} T^{n}\left(I-T T^{*}\right)^{1 / 2} T+\left(I-T^{*} T\right)^{1 / 2} T^{n}\left(I-T T^{*}\right) \\
& \times\left(I-T^{*}\right)^{-1}\left(I-T^{*} T\right)^{1 / 2} \\
& =-\left(I-T^{*} T\right)^{1 / 2} T^{n+1}\left(I-T^{*} T\right)^{1 / 2} \\
& +\left(I-T^{*} T\right)^{1 / 2} T^{n}\left(I-T^{*}\right)^{-1}\left(I-T^{*} T\right)^{1 / 2} \\
& -\left(I-T^{*} T\right)^{1 / 2} T^{n+1} T^{*}\left(I-T^{*}\right)^{-1}\left(I-T^{*} T\right)^{1 / 2} \\
& \text { (7) }=-\left(I-T^{*} T\right)^{1 / 2} T^{n+1}\left(I-T^{*} T\right)^{1 / 2} \\
& +\left(I-T^{*} T\right)^{1 / 2} T^{n}\left(I-T^{*}\right)^{-1}\left(I-T^{*} T\right)^{1 / 2} \\
& -\left(I-T^{*} T\right)^{1 / 2} T^{n+1}\left(I-T^{*}\right)^{-1}\left(I-T^{*} T\right)^{1 / 2} \\
& +\left(I-T^{*} T\right)^{1 / 2} T^{n+1}\left(I-T^{*} T\right)^{1 / 2} \\
& =\left(I-T^{*} T\right)^{1 / 2} T^{n}\left(I-T^{*}\right)^{-1}\left(I-T^{*} T\right)^{1 / 2} \\
& -\left(I-T^{*} T\right)^{1 / 2} T^{n+1}\left(I-T^{*}\right)^{-1}\left(I-T^{*} T\right)^{1 / 2} .
\end{aligned}
$$

Thus we have from (6) and (7) that:

Proposition 5. Suppose that $\|T\| \leqq 1, r(T)<1$. Then $U_{T}$ is scalar 
if and only if $\left\{\left(I-T^{*} T\right)^{1 / 2} T^{n}\left(I-T^{*}\right)^{-1}\left(I-T^{*} T\right)^{1 / 2}: n \geqq 0\right\}$ is a commuting family of normal operators.

A useful sufficient set of conditions is then immediate.

Proposition 6. Suppose that $\|T\| \leqq 1, r(T)<1$, and

$$
\left\{\left(I-T^{*} T\right)^{1 / 2} T^{n} T^{* m}\left(I-T^{*} T\right)^{1 / 2}: n \geqq 0, m \geqq 0\right\}
$$

is a commuting family of normal operators. Then $U_{T}$ is scalar.

The conditions of Proposition 6 are somewhat easier to work with than those of 5 . It seems resonable to conjecture that Proposition 6 gives necessary as well as sufficient conditions for $U_{T}$ to be scalar, but to date we have not been able to prove it.

6. Examples. In this section we will explicitly construct some operators $T$ such that $U_{T}$ is scalar. The construction using operators in $\Theta$ will be given later.

Recall that an operator $T$ on $h$ is called $n$-normal if $h$ can be considered as an orthogonal sum of $n$-copies of a Hilbert space $h_{0}$ and relative to this decomposition $T$ can be written as an $n \times n$ matrix whose entries $A_{i j}$ are all normal operators which commute with each other.

We will also need the following lemma. We omit its proof.

LEMMA 1. If $X, X Y X$ are commuting normal operators and $X$ is one-to-one, then $Y$ is normal and $[X, Y]=0$.

Suppose that $\|T\| \leqq 1, r(T)<1$, and $U_{T}$ is scalar. Relative to the decomposition $h=R\left(I-T^{*} T\right) \oplus N\left(I-T^{*} T\right), T$ has the operator matrix

$$
\begin{aligned}
T= & {\left[\begin{array}{ll}
A & B \\
C & E
\end{array}\right], \text { where } } \\
& B^{*} B+E^{*} E=I, \\
& A^{*} B+C^{*} E=0,
\end{aligned}
$$

and $I-A^{*} A-C^{*} C=D_{0}, D_{0} \geqq 0$, is one-to-one. Note that

$$
\left(I-T^{*} T\right)=\left[\begin{array}{ll}
D_{0} & 0 \\
0 & 0
\end{array}\right] .
$$


From Proposition 6 we have

Proposition 7. If the $A, B, C, E$ in (8) are commuting normal operators, then $T=\left[\begin{array}{ll}A & B \\ C & E\end{array}\right]$ is such that $U_{T}$ is scalar provided that $r(T)<1$.

In building an example, $T$ will be nonnormal if $B B^{*} \neq C^{*} C$ or $A C^{*}+B E^{*} \neq 0$ or $C C^{*}+E E^{*} \neq I$. One way to get $r(T)<1$ is to have $\|A+E\|+\|A\|+\|C\|<1$. It is an immediate consequence of Lemma 1 that:

Proposition 8. The $A$ in the block form (8) of $T$ is normal if $U_{T}$ is scalar.

However, it is easy to construct $T$ such that $U_{T}$ is scalar and $T$ in the block form (8) is not $n$-normal.

EXAMPLE 1. Let $h=h_{0} \oplus h_{0}$ and let $S$ be a unilateral shift on $h_{0}$. Define $T_{\delta}$ on $h$ by

$$
T_{\delta}=\left[\begin{array}{cc}
\delta I & 1 / \sqrt{2} S \\
-\delta S^{*} & 1 / \sqrt{2} I
\end{array}\right], 0 \leqq \delta<1 .
$$

Then $T_{\delta}$ is in the block form (8). $\quad T_{\delta} \rightarrow T_{0}$ uniformly as $\delta \rightarrow 0$. But $r\left(T_{0}\right)=1 / \sqrt{2}<1$ so $r\left(T_{\delta}\right)<1$ for sufficiently small $\delta$. That $U_{T_{\delta}}$ is scalar for $r\left(T_{\delta}\right)<1$ follows from Proposition 6 .

Finally we note that if $\operatorname{dim} R\left(I-T^{*} T\right)>1$ and $U_{T}$ is scalar, then $T$ has reducing subspaces by Theorem 1 and Proposition 3.

7. The case $r(T)=1$. If $r(T)=1, T^{n} \rightarrow 0$ strongly, and $T^{*}$ is not an isometry, then $V_{T}$ exists and has the formula (1). Suppose $V_{T} U_{0}$ is scalar for some constant unitary $U_{0}$. Proposition 2 does not necessarily apply since $\left\{V_{T}(w) U_{0}:|w|=1\right\}$ is only a commuting family of unitary operators for almost all $w$. However, this difficulty is easily avoided. Suppose $|\alpha|=1$. Then $V_{\alpha T}(w)=V_{T}(\alpha w) \bar{\alpha}$. Thus by replacing $T$ by $\alpha T$ for a suitable $\alpha$, we get that Proposition 2 holds for $\alpha T$.

In trying to duplicate the results of $\S 5$ one has to use the series (1) since $(I-w T)$ does not have a bounded inverse for all $|w|=1$. This presents difficulties in calculating $U_{T}$. The proof of Proposition 5 relies on the fact that $\left(I-T T^{*}\right)^{1 / 2}$ can be factored out of $\Sigma_{n=0}^{\infty}\left(I-T T^{*}\right)^{1 / 2} T^{* n}$. If $\alpha$ can be chosen so that $|\alpha|=1, \alpha \notin \sigma(T)$, then one can get Proposition 5 
to hold for $\bar{\alpha} T$. If $\{w:|w|=1\} \subseteq \sigma(T)$, then the calculations would have to be modified. We will not attempt them here.

8. An application. There is another way to "build" scalar inner functions. It was part of our original reason for undertaking this study. Recall that we let $\Theta$ denote $\left\{T:\left[T^{*} T, T+T^{*}\right]=0\right\}$. If $Q$ is quasinormal $\left(\left[Q, Q^{*} Q\right]=0\right), A$ is self-adjoint, and $[A, Q]=0$, then $A+Q \in \Theta$. Whether there exists other types of operators in $\Theta$ is an open question. The defining condition of $\Theta$ occurs, for example, in [5]. It would be of interest to characterize the operators in $\Theta$.

Suppose that $\|T\|<1$ and form the inner function $V=$ $V_{T} V_{T^{*}} \quad V \in(A I)$ since $V_{T}, V_{T^{*}} \in(A I)$. From (3) we have that

$$
\begin{gathered}
V(w)=\left(I-T^{*} T\right)^{-1 / 2}\left(w I-T^{*}\right)(I-w T)^{-1}(w I-T) \\
\times\left(I-w T^{*}\right)^{-1}\left(I-T^{*} T\right)^{1 / 2} \\
=\left(I-T^{*} T\right)^{-1 / 2}\left(w I-T^{*}\right)\left(\begin{array}{r}
w I-T) \\
\times(I-w T)^{-1}\left(I-w T^{*}\right)^{-1}\left(I-T^{*} T\right)^{1 / 2} \\
=\left(I-T^{*} T\right)^{-1 / 2}\left(T^{*} T-w\left(T^{*}+T\right)+w^{2} I\right) \\
\times\left(I-w\left(T^{*}+T\right)+w^{2} T^{*} T\right)^{-1}\left(I-T^{*} T\right)^{1 / 2} .
\end{array}\right.
\end{gathered}
$$

If $T \in \Theta$, then $V$ would be scalar. Now $H_{\hbar}^{2} \ominus V_{T} H_{h}^{2} \subseteq H_{h}^{2} \ominus V H_{h}^{2}$ since $V=V_{T} V_{T^{*}}$. We have then that $T$ is unitarily equivalent to the restriction of an operator with scalar inner function to an invariant subspace. Thus information about operators associated with scalar inner functions might prove useful in analyzing the operators in $\theta$. Conversely, finding additional operators in $\Theta$ allows us to construct additional examples of scalar inner functions.

We will not go into a detailed study of $T \in \Theta$, but will make a few basic observations. The proofs of the first two are trivial, but the propositions are often useful when working with operators in $\Theta$.

Proposition 9. $T \in \Theta$ if and only if $T^{*}\left[T^{*}, T\right]=\left[T^{*}, T\right] T$.

Proposition 10. If $T \in \Theta$, then $N(T)$ is reducing.

THEOREM 3. If $T \in \Theta$ and $T$ is a trace class compact operator, then $T$ is normal.

Proof. Suppose that $T \in \Theta$ and $T$ is a trace class compact operator. Then

$$
\left[T^{*}, T\right]^{2}=T^{*} T T^{*} T-T^{*} T^{2} T^{*}-T T^{* 2} T+T T^{*} T T^{*}
$$


Thus,

$$
\begin{aligned}
\frac{1}{2} \operatorname{trace}\left(\left[T^{*}, T\right]^{2}\right) & =\operatorname{trace}\left(\left(T^{*} T\right)^{2}\right)-\operatorname{trace}\left(T^{* 2} T^{2}\right) \\
& =\operatorname{trace}\left(\left(T^{*} T\right)^{2}-T^{* 2} T^{2}\right) \\
& =-\operatorname{trace}\left(T^{*}\left[T^{*}, T\right] T\right) \\
& =-\operatorname{trace}\left(\left[T^{*}, T\right] T^{2}\right) \\
& =-\operatorname{trace}\left(T^{*} T^{3}-T T^{*} T^{2}\right) \\
& =-\operatorname{trace}\left(T^{*} T^{3}\right)+\operatorname{trace}\left(T^{*} T^{3}\right)=0 .
\end{aligned}
$$

But $\left[T^{*} T\right]^{2} \geqq 0$, thus $\left[T^{*} T\right]=0$ and $T$ is normal.

A discussion of trace class operators may be found in $[4, \mathrm{pp}$. 1088-1119].

Note that if $\operatorname{dim} h<\infty$, then Theorem 3 says that $T \in \Theta$ if and only if $T$ is normal.

We shall now examine the relationship between $V$ and $T$ more closely. Our first two results, while dealing with basic uniqueness properties of Potapov inner functions, are apparently new.

Assume for the remainder of this paper that $T \in \Theta$ and $\|T\|<$ 1. Then $V=V_{T} V_{T^{*}}$ is scalar. Let $\tilde{T}$ denote $S^{*}$ restricted to $H_{\hbar}^{2} \ominus V H_{h}^{2}$. Then $\|\tilde{T}\| \leqq 1, r(\tilde{T})<1$ since $V \in(A I) . \quad V$ is scalar so that $V(w)=\int b(w, \lambda) E(d \lambda)$. If $c(w, \lambda),|w|=1$, is an inner function that divides $b(w, \lambda)$ almost everywhere and $c(\cdot, \cdot)$ is a borel measurable function, then $V_{1}(w)=\int c(w, \lambda) E(d \lambda)$ defines a scalar inner function that divides $V$ [9]. Inner functions like $V_{1}$ are the obvious factors for a scalar $V$. Note that $\left[V, V_{1}\right]=0 . \quad V_{T}$ is also a factor of $V$ but neither $V_{T}$ nor $U_{T}$ are scalar if $T$ is nonnormal. Thus $V_{T}$ is a nontrivial example of a nonscalar factor of a scalar inner function.

Now if $\|\tilde{T} f\|<\|f\|$ for all nonzero $f \in H_{\hbar}^{2} \ominus V H_{h}^{2}$, then $V=V_{\tilde{T}} U_{0}$ for some $\tilde{T}_{0}$ acting in $h$ and a unitary $U_{0}[1] . \quad \tilde{T}_{0}$ is unitarily equivalent to $\tilde{T}$. $\tilde{T}_{0}$ would be normal since $V$ is scalar [3], and $T$ would have to be subnormal. Since it is unknown if all the operators in $\Theta$ are subnormal, it becomes important to determine if $\|\tilde{T} f\|<\|f\|$ for all nonzero $f$.

It follows from a result of Virot [11] about Rota inner functions that:

THEOREM 4. If $T_{1}, T_{2}$ are operators of norm less than one, and $V_{T_{1}} H_{h}^{2} \subseteq V_{T_{2}} H_{h}^{2}$, then $T_{1}=T_{2}$.

Proof. Since $\left\|T_{1}\right\|<1,\left\|T_{2}\right\|<1$, there exists $T_{3}, T_{4}$ such that $r\left(T_{3}\right)$, $r\left(T_{4}\right)<1$ and $V_{T_{1}}=R_{T_{3}} U_{1}, V_{T_{2}}=R_{T_{4}} U_{2}$ where $R_{T_{i}}$ is the Rota inner 
function of $T_{i}$ and $U_{i}$ is a constant unitary operator [8, p. 29]. But Virot has shown that $T_{3}=T_{4}$ if $R_{T_{3}} H_{h}^{2} \subseteq R_{T_{4}} H_{h}^{2}$. Hence $V_{T_{1}} H_{h}^{2}=$ $V_{T_{2}} H_{h}^{2}$. Thus $V_{T_{1}}=V_{T_{2}} U_{0}$ for some constant unitary $U_{0}$. Evaluation at $w=0$ gives $T_{1}^{*}=T_{2}^{*} U_{0}$, so that $\left(I-T_{1}^{*} T_{1}\right)=\left(I-T_{2}^{*} T_{2}\right)$, and $\left(I-T_{1} T_{1}^{*}\right)=U_{0}^{*}\left(I-T_{2} T_{2}^{*}\right) U_{0}$. But the $w$ terms of $V_{T_{1}}$ and $V_{T_{2}} U_{0}$ must be equal. That is,

$$
\left(I-T_{1}^{*} T_{1}\right)^{1 / 2}\left(I-T_{1} T_{1}^{*}\right)^{1 / 2}=\left(I-T_{2}^{*} T_{2}\right)^{1 / 2}\left(I-T_{2} T_{2}^{*}\right)^{1 / 2} U_{0} .
$$

But $\left(I-T_{i}^{*} T_{i}\right)^{1 / 2},\left(I-T_{\imath} T_{\imath}^{*}\right)^{1 / 2}$ are one-to-one. Thus

$$
U_{0}^{*}\left(I-T_{2} T_{2}^{*}\right)^{1 / 2} U_{0}=\left(I-T_{1} T_{1}^{*}\right)^{1 / 2}=\left(I-T_{2} T_{2}^{*}\right)^{1 / 2} U_{0}
$$

and $U_{0}=I$. Hence $T_{1}=T_{2}$.

The last half of the proof of Theorem 4 is of some interest in its own right. It shows that:

Proposition 11. If $\left\|T_{1} \phi\right\|<\|\phi\|$ for all nonzero $\phi \in h$ and if $V_{T_{1}} H_{\hbar}^{2}=V_{T_{2}} H_{\hbar}^{2}$, then $T_{1}=T_{2}$.

The assumption on $T_{1}$ in Proposition 11 is needed.

EXAMPLE 2. Let

$$
T_{1}=\left[\begin{array}{ll}
0 & 1 \\
0 & 0
\end{array}\right], \quad T_{2}=\left[\begin{array}{ll}
0 & \alpha \\
0 & 0
\end{array}\right], \quad \text { and } \quad U_{0}=\left[\begin{array}{cc}
\bar{\alpha} & 0 \\
0 & \alpha
\end{array}\right]
$$

where $|\alpha|=1, \alpha \neq 1$. Then

$$
V_{T_{1}}(w)=\left[\begin{array}{cc}
0 & w^{2} \\
-1 & 0
\end{array}\right] \text { and } V_{T_{2}}(w)=\left[\begin{array}{ll}
0 & \alpha w^{2} \\
-\bar{\alpha} & 0
\end{array}\right]
$$

Thus $V_{T_{1}} U_{0}=V_{T_{2}}$ so that $V_{T_{1}} H_{h}^{2}=V_{T_{2}} H_{h}^{2}$ but $T_{1} \neq T_{2} . \quad T_{1}$, of course, is unitarily equivalent to $T_{2}$. Note that in this example $r\left(T_{i}\right)<1,\left\|T_{i}^{2}\right\|<1$ also.

Example 2 also shows that the hypothesis of Theorem 4 cannot be weakened to $r\left(T_{i}\right)<1$.

Since $V H_{\hbar}^{2} \subseteq V_{T} H_{\hbar}^{2}$, we have $\|\tilde{T}\|=1$ otherwise Theorem 4 would give us that $V H_{\hbar}^{2}=V_{T} H_{\hbar}^{2}$ which is a contradiction. We shall now show that there is an $f$ such that $\|\tilde{T} f\|=\|f\|$. The proof will depend on the fact that if $\|X\| \leqq 1, r(X)<1$, then 


$$
H_{h}^{2} \ominus V_{X} H_{h}^{2}=\left\{\left(I-X^{*} X\right)^{1 / 2}(I-w X)^{-1} \phi: \phi \in h\right\}
$$

Proposition 12. Supposé that $T \in \Theta,\|T\|<1$. Let $V=V_{T} V_{T^{*}}$ and $\tilde{T}$ be $S^{*}$ acting in $H_{h}^{2} \ominus V H_{h}^{2}$. Then $\|\tilde{T} f\|=\|f\|$ for some $f \in H_{\hbar}^{2} \ominus V H_{\hbar}^{2}$.

Proof. Since $V H_{\hbar}^{2} \subseteq V_{T} H_{\hbar}^{2}$ we have

$$
H_{h}^{2} \ominus V H_{h}^{2}=\left(H_{h}^{2} \ominus V_{T} H_{h}^{2}\right) \oplus\left(V_{T} H_{h}^{2} \ominus V_{T} V_{T} \cdot H_{h}^{2}\right) .
$$

Suppose that $f \in H_{h}^{2} \ominus V H_{h}^{2}$. Then from (10) we have $f=f_{1} \oplus V_{T} f_{2}$ where $f_{1} \in H_{h}^{2} \ominus V_{T} H_{h}^{2}$ and $f_{2} \in H_{h}^{2} \ominus V_{T} \cdot H_{h}^{2}$. Thus there are $\phi, \psi \in h$ such that $f_{1}=\left(I-T^{*} T\right)^{1 / 2}(I-w T)^{-1} \phi$ and

$$
f_{2}=\left(I-T T^{*}\right)^{1 / 2}\left(I-w T^{*}\right)^{-1} \psi
$$

$\|\tilde{T} f\|=\|f\|$ if and only if $f(0)=0$. But

$$
f(0)=\left(I-T^{*} T\right)^{1 / 2} \phi-T^{*}\left(I-T T^{*}\right)^{1 / 2} \psi=\left(I-T^{*} T\right)^{1 / 2}\left(\phi-T^{*} \psi\right) .
$$

Hence $\|\tilde{T} f\|=\|f\|$ whenever $\phi=T^{*} \psi$.

Thus $V$ is the scalar type of operator discussed in the earlier sections of this paper.

We shall conclude by calculating explicitly the relationship between $\tilde{T}$ and $T$. This will enable us to write down a nonnormal $T^{\prime}$ such that $U_{T^{\prime}}$ is scalar for any $T \in \Theta,\|T\|<1$. If $T \in \Theta$ is normal, $T^{\prime}$ will be a nonnormal 2-normal operator.

By Theorem 3 we may assume $\operatorname{dim} h$ is infinite. Let $h=h_{1} \oplus h_{2}$ where $\operatorname{dim} h=\operatorname{dim} h_{1}=\operatorname{dim} h_{2}$. Let $E_{i}$ be an isometry from $h$ onto $h_{\imath}$. Thus if $\phi \in h$, we have

$$
\phi=E_{1} \phi_{1} \oplus E_{2} \phi_{2} \text { for unique } \phi_{1}, \phi_{2} \in h .
$$

Define $B$ sending $h$ onto $H_{h}^{2} \ominus V H_{h}^{2}$ by

$$
B \phi=\left(I-T^{*} T\right)^{1 / 2}(I-w T)^{-1} \phi_{1} \oplus V_{T}(w)\left(I-T T^{*}\right)^{1 / 2}\left(I-w T^{*}\right)^{-1} \phi_{2}
$$

where $\phi_{1}, \phi_{2}$ are defined by (11). The sum in (12) is the same orthogonal sum as in (10). It is clear from (11) and (12) that $B$ is a one-to-one continuous linear transformation. From $(10)$ we get that $B$ is an isometry. Following [8] we define $\hat{T}$ as follows. If $\phi \in h$, then $B \phi \in H_{h}^{2} \ominus V H_{h}^{2}$. Thus $S^{*} B \phi=\tilde{T} B \phi \in H_{h}^{2} \ominus V H_{h}^{2}$. Hence there is a $\psi \in h$ such that $\tilde{T} B \phi=B \psi$. Define $\hat{T} \phi=\psi$. We have then that $\tilde{T} B=B \hat{T}$ and $\hat{T}$ is unitarily equivalent to $\tilde{T}$. Recall that $D=$ 
$\left(I-T^{*} T\right)^{1 / 2}$. Let $D_{*}=\left(I-T T^{*}\right)^{1 / 2}$. Take $\phi=E_{1} \phi_{1}$ so that $B \phi=$ $D(I-w T)^{-1} \phi_{1}$. Then $\tilde{T} B \phi=D(I-w T)^{-1} T \phi_{1}=B \psi \quad$ where $\quad \psi=$ $E_{1} T \phi_{1}$. Thus $\hat{T} E_{1} \phi=E_{1} T \phi_{1}$. That is, $h_{1}$ is an invariant subspace for $\hat{T}$ and $\tilde{T}$ restricted to $h_{1}$ is unitarily equivalent to $T$ on $h$.

Relative to the decomposition $h=h_{1} \oplus h_{2}$ we have

$$
\hat{T}=\left[\begin{array}{cc}
E_{1} T E_{1}^{*} & X \\
0 & Y
\end{array}\right] .
$$

We now determine the two terms $X, Y$. Take $\phi=E_{2} \phi_{2}$ so that

$$
B \phi=V_{T}(w) D_{*}\left(I-w T^{*}\right)^{-1} \phi_{2} .
$$

Then

$$
\begin{aligned}
\tilde{T} B \phi & =S^{*}\left\{V_{T}(w) D_{*}\left(w T^{*}\left(I-w T^{*}\right)^{-1}+I\right)\right\} \phi_{2} \\
& =S^{*}\left\{V_{T}(w) D_{*} \phi_{2}\right\}+V_{T}(w) D_{*}\left(I-w T^{*}\right)^{-1} T^{*} \phi_{2}
\end{aligned}
$$

Notice that $V_{T}(w) D_{*}\left(I-w T^{*}\right)^{-1} T^{*} \phi_{2} \in V_{T} H_{\hbar}^{2} \ominus V H_{\hbar}^{2}$. We shall show that $S^{*}\left\{V_{T}(w) D_{*} \phi_{2}\right\} \in H_{\hbar}^{2} \ominus V_{T} H_{h}^{2}$. Since $\|T\|<1$, we have

$$
\begin{aligned}
S^{*}\left\{V_{T}(w) D_{*} \phi_{2}\right\} & =S^{*}\left\{D^{-1}\left(w I-T^{*}\right)(I-w T)^{-1} D_{*} D_{*} \phi_{2}\right\} \\
& =S^{*}\left\{D^{-1}\left[w(I-w T)^{-1}-T^{*}(I-w T)^{-1}\right] D_{*}^{2} \phi_{2}\right\} \\
& =D^{-1}\left[(I-w T)^{-1}-T^{*} T(I-w T)^{-1}\right] D_{*}^{2} \phi_{2} \\
& =D^{-1}\left(I-T^{*} T\right)(I-w T)^{-1} D_{*}^{2} \phi_{2} \\
& =D(I-w T)^{-1} D_{*}^{2} \phi_{2}
\end{aligned}
$$

which is in $H_{h}^{2} \ominus V_{T} H_{h}^{2}$. Thus

$$
\hat{T} E_{2} \phi_{2}=E_{1}\left(I-T T^{*}\right) \phi_{2}+E_{2} T^{*} \phi_{2}
$$

or

$$
\hat{T}=\left[\begin{array}{cc}
E_{1} T E_{1}^{*} & E_{1}\left(I-T T^{*}\right) E_{2}^{*} \\
0 & E_{2} T^{*} E_{2}^{*}
\end{array}\right] .
$$

We have the following theorem.

TheOREM 5. Suppose that $\left[T^{*} T, T+T^{*}\right]=0,\|T\|<1$, and let $V=V_{T} V_{T^{*}}$. Then $V$ is scalar. Furthermore $S^{*}$ restricted to $H_{h}^{2} \ominus V H_{h}^{2}$ is unitarily equivalent to 


$$
T^{\prime}=\left[\begin{array}{cc}
T & \left(I-T T^{*}\right) \\
0 & T^{*}
\end{array}\right]
$$

acting in $h \oplus h$. The operator $T^{\prime}$ is such that $\left\|T^{\prime}\right\|=1,1 \in \sigma_{p}\left(T^{*} T^{\prime}\right)$, $r\left(T^{\prime}\right)<1$, and $U_{T^{\prime}}$ is scalar.

Proof. The only part of the theorem that needs to be verified is that $U_{T^{\prime}}$ is scalar. But from the proof of Theorem 2 of [1] it is clear that if we write $V=c V_{X} \tau U_{1}$ as in (5), then $X$ is unitarily equivalent to $T^{\prime}$. Thus $U_{T^{\prime}}$ is scalar by Corollary 1 .

Unfortunately $T^{\prime}$ does not help answer the question of whether $T \in \Theta$ implies $T$ is subnormal. Neither $T^{\prime}$ nor $T^{* *}$ are even hyponormal since $\left\|T^{\prime}\right\|>r\left(T^{\prime}\right)$.

Acknowledgement. The author would like to thank Carl D. Meyer, Jr. for the proof of Theorem 3 that appears here.

\section{REFERENCES}

1. Stephen L. Campbell, Operator valued inner functions analytic on the closed disc, Pacific J. Math., 41 (1972), 57-62.

2. - Inner functions analytic at a point, Ill. J. Math., 16 (1972), 651-652.

3. Commutation properties of the coefficient matrix in the differential equation of an inner function, Proc. Amer. Math. Soc., 42 (1974), 507-512.

4. N. Dunford and J. Schwartz, Linear Operators, Part II, Interscience, New York, 1963.

5. Mary R. Embry, Conditions implying normality in Hilbert space, Pacific J. Math., 18 (1966), 457-460.

6. Henry Helson, Lectures on Invariant Subspaces, Academic Press, New York, 1964.

7. - The differential equation of an inner function, Studia Math., 35 (1970), 311-321.

8. S. Lynn Jackson, Jr., Operators and Inner Functions, Dissertation at Univ. of California, Berkeley, 1967.

9. Malcolm J. Sherman, A spectral theory for inner functions, Trans. Amer. Math. Soc., 135 (1969), 387-398.

10. Sheldon O. Sickler, The invariant subspaces of almost unitary operators, preprint.

11. Bernard Virot, Sur le modèle de Rota d'une contraction stricte d'un espace de Hilbert complexe séparable, C. R. Acad. Sc. Paris, Série A, t., 269 (1969), 130-133.

Received May 16, 1974.

North Carolina State University

Raleigh, North Carolina, 27607 



\section{PACIFIC JOURNAL OF MATHEMATICS}

\section{EDITORS}

RICHARD ARENS (Managing Editor)

University of California

Los Angeles, California 90024

\author{
R. A. Beaumont \\ University of Washington \\ Seattle, Washington 98105
}

\section{J. DugundII}

Department of Mathematics

University of Southern California

Los Angeles, California 90007

D. Gilbarg and J. Milgram

Stanford University

Stanford, California 94305

\section{ASSOCIATE EDITORS}
E. F. BECKENBACH
B. H. NeumanN
F. WoLF
K. YoshidA

\section{SUPPORTING INSTITUTIONS}

\author{
UNIVERSITY OF BRITISH COLUMBIA \\ CALIFORNIA INSTITUTE OF TECHNOLOGY \\ UNIVERSITY OF CALIFORNIA \\ MONTANA STATE UNIVERSITY \\ UNIVERSITY OF NEVADA \\ NEW MEXICO STATE UNIVERSITY \\ OREGON STATE UNIVERSITY \\ UNIVERSITY OF OREGON \\ OSAKA UNIVERSITY
}

\author{
UNIVERSITY OF SOUTHERN CALIFORNIA \\ STANFORD UNIVERSITY \\ UNIVERSITY OF TOKYO \\ UNIVERSITY OF UTAH \\ WASHINGTON STATE UNIVERSITY \\ UNIVERSITY OF WASHINGTON \\ AMERICAN MATHEMATICAL SOCIETY
}

The Supporting Institutions listed above contribute to the cost of publication of this Journal, but they are not owners or publishers and have no responsibility for its contents or policies.

Mathematical papers intended for publication in the Pacific Journal of Mathematics should be in typed form or offset-reproduced (not dittoed), double spaced with large margins. Underline Greek letters in red, German in green, and script in blue. The first paragraph or two must be capable of being used separately as a synopsis of the entire paper. Items of the bibliography should not be cited there unless absolutely necessary, in which case they must be identified by author and Journal, rather than by item number. Manuscripts, in duplicate, may be sent to any one of the four editors. Please classify according to the scheme of Math. Reviews, Index to Vol. 39. All other communications should be addressed to the managing editor, or Elaine Barth, University of California, Los Angeles, California, 90024.

100 reprints are provided free for each article, only if page charges have been substantially paid. Additional copies may be obtained at cost in multiples of 50.

The Pacific Journal of Mathematics is issued monthly as of January 1966. Regular subscription rate: $\$ 72.00$ a year (6 Vols., 12 issues). Special rate: $\$ 36.00$ a year to individual members of supporting institutions.

Subscriptions, orders for back numbers, and changes of address should be sent to Pacific Journal of Mathematics, 103 Highland Boulevard, Berkeley, California, 94708.

PUBLISHED BY PACIFIC JOURNAL OF MATHEMATICS, A NON-PROFIT CORPORATION Printed at Jerusalem Academic Press, POB 2390, Jerusalem, Israel.

\section{Copyright (C) 1975 Pacific Journal of Mathematics All Rights Reserved}




\section{Pacific Journal of Mathematics

Vol. 60, No. $2 \quad$ October, 1975

Waleed A. Al-Salam and A. Verma, A fractional Leibniz q-formula ........... 1

Robert A. Bekes, Algebraically irreducible representations of $L_{1}(G) \ldots \ldots \ldots \ldots 11$

Thomas Theodore Bowman, Construction functors for topological

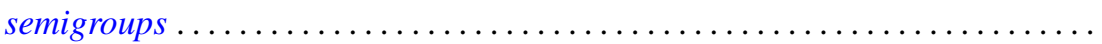

Stephen LaVern Campbell, Operator-valued inner functions analytic on the

closed disc. II .........................................

Leonard Eliezer Dor and Edward Wilfred Odell, Jr., Monotone bases in $L_{p} \ldots \ldots$.

Yukiyoshi Ebihara, Mitsuhiro Nakao and Tokumori Nanbu, On the existence of

global classical solution of initial-boundary value problem for

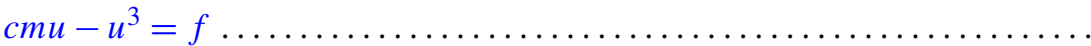

Y. Gordon, Unconditional Schauder decompositions of normed ideals of

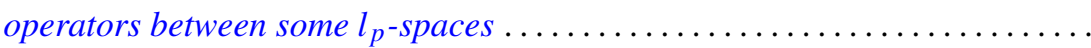

Gary Grefsrud, Oscillatory properties of solutions of certain nth order functional

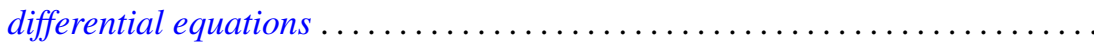

Irvin Roy Hentzel, Generalized right alternative rings ...................

Zensiro Goseki and Thomas Benny Rushing, Embeddings of shape classes of compacta in the trivial range .................................

Emil Grosswald, Brownian motion and sets of multiplicity . .

Donald LaTorre, A construction of the idempotent-separating congruences on a

bisimple orthodox semigroup .

Pjek-Hwee Lee, On subrings of rings with involution ...

Marvin David Marcus and H. Minc, On two theorems of Frobenius ...

Michael Douglas Miller, On the lattice of normal subgroups of a direct

product. .

Grattan Patrick Murphy, A metric basis characterization of Euclidean space

Roy Martin Rakestraw, A representation theorem for real convex functions ....

Louis Jackson Ratliff, Jr., On Rees localities and $H_{i}$-local rings ...

Simeon Reich, Fixed point iterations of nonexpansive mapping . .

Domenico Rosa, $B$-complete and $B_{r}$-complete topological algebras ...

Walter Roth, Uniform approximation by elements of a cone of real-valued

functions ....

Helmut R. Salzmann, Homogene kompakte projektive Ebenen

Jerrold Norman Siegel, On a space between $B H$ and $B_{\infty} \ldots$

235

Robert C. Sine, On local uniform mean convergence for Markov operators

James D. Stafney, Set approximation by lemniscates and the spectrum of an

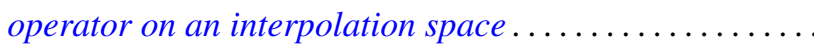

Árpád Száz, Convolution multipliers and distributions .......

Kalathoor Varadarajan, Span and stably trivial bundles ..........

Robert Breckenridge Warfield, Jr., Countably generated modules over

commutative Artinian rings....................... 